INPLASY

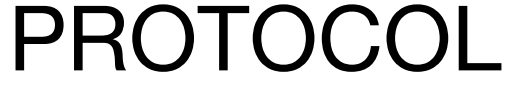

To cite: Fernandez-Rio et al.

Cooperative learning in

teacher education: A

systematic review. Inplasy

protocol 202180099. doi:

10.37766/inplasy2021.8.0099

Received: 25 August 2021

Published: 25 August 2021

Corresponding author:

Damián Iglesias

diglesia@unex.es

Author Affiliation:

Teacher Training College.

University of Extremadura.

Spain.

Support: None.

Review Stage at time of this submission: Data analysis.

Conflicts of interest:

None declared.

\section{Cooperative learning in teacher education: A systematic review}

Fernandez-Rio, J1; Rivera-Pérez, S2; Iglesias, D³.

Review question / Objective: What do we know about the impact of cooperative learning (CL) interventions on future teachers so far? More specifically, this review aimed to answer the following research questions: (RQ1) What was the focus of studies investigating the benefits of $C L$ on teacher education? (RQ2) Which learning outcomes have been observed? (RQ3) What does literature indicate are the main effects of CL on teacher education students?

Condition being studied: Effects promoted by cooperative learning in teacher education.

Information sources: Several electronic literature databases were involved in the process (Web of Science, EBSCO host, Scopus, Eric, PsycINFO, SciELO).

INPLASY registration number: This protocol was registered with the International Platform of Registered Systematic Review and Meta-Analysis Protocols (INPLASY) on 25 August 2021 and was last updated on 25 August 2021 (registration number INPLASY202180099).

\section{INTRODUCTION}

Review question / Objective: What do we know about the impact of cooperative learning (CL) interventions on future teachers so far? More specifically, this review aimed to answer the following research questions: (RQ1) What was the focus of studies investigating the benefits of CL on teacher education? (RQ2) Which learning outcomes have been observed? (RQ3) What does literature indicate are the main effects of CL on teacher education students? 
Condition being studied: Effects promoted by cooperative learning in teacher education.

\section{METHODS}

Participant or population: Student-teachers of any ethnicity or country engaging in regular universities.

Intervention: Cooperative learning.

Comparator: Control, equivalent or comparison groups.

Study designs to be included: The impact of cooperative learning interventions on future teachers.

Eligibility criteria: Empirical studies met the following inclusion criteria: (1) Peerreviewed journal articles indexed in the Journal Citation Report (JCR) or the Scimago Journal Rank (SJR) published and written in English from January 2001 to December 2020, (2) CL interventions including fundamental CL elements, CL structures (e.g., jigsaw) or similar cooperative teaching strategies, (3) Quantitative, qualitative or mixed intervention studies, (4) Teacher education in a university degree that enables graduates to teach in the different educational stages prior to the university.Upon selection of the articles, researchers ensured that the studies met the "Population, Intervention, Comparison and Outcome" (PICO) standards (Liberati et al., 2009). Population: student-teachers of any ethnicity or country engaging in regular universities. Intervention: studies that assessed effects and reported the impact on learning outcomes. Comparison: learning outcomes were assessed postintervention and/or compared with control, equivalent or comparison groups. Output: the studies identified changes in learning outcomes. Exclusion criteria were: (1) Cross-sectional designs, (2) Group or team work studies without guaranteeing the fidelity of a CL-based intervention, (3) Combining $\mathrm{CL}$ with other teaching strategies, (4) Other university degrees (e.g., law, engineering, veterinary) (5)
Implementations conducted in courses, seminars or other short formats outside the university context.

Information sources: Several electronic literature databases were involved in the process (Web of Science, EBSCO host, Scopus, Eric, PsycINFO, SciElO). A combination of keywords and filters were used with the Boolean logic-commands "AND" and "OR". Searches included article titles, abstracts and keywords by following this query string: [("cooperative learning" OR "collaborative learning") AND ("preservice teacher"” OR "prospective teacher*" OR "student* teacher*" OR "future teacher" OR "teacher education" OR "higher education" OR "teacher* training") AND ("intervention*" OR "program"” OR "effect" OR "experimental group" OR "control group" OR "comparison group")]. No previous research addressed a systematic review on this topic, so to get a contemporary picture of the field, a 20-year reviewing period was selected.

Main outcome(s): Content knowledge, pedagogical content knowledge, personal, inter-personal and transversal skills.

Quality assessment / Risk of bias analysis: It was based on the following criteria: (a) description of the program; (b) JCR/SJR journal; (c) detailed methodological description; (d) sample or number of participants; and (e) length of the implementation. Each item was scored from 0 to 2 using the criteria described in Table 2. A total quality score for each one of the selected publications was calculated by adding all the scores. Finally, studies were classified as: (a) low quality: score lower than 3; (b) moderate quality: score between 4 and 6; and (c) high quality: score of 7 or more.

Strategy of data synthesis: A summary of scope and findings reported of the studies (author, year, country, participants, interventions, duration, outcomes, conclusions).

Subgroup analysis: No analysis. 
Sensitivity analysis: No analysis.

Language: English.

Country(ies) involved: Spain.

Keywords: Cooperative Learning; Teacher

Education; Review.

Contributions of each author:

Author 1 - Javier Fernandez-Rio.

Email: javier.rio@uniovi.es

Author 2 - Sergio Rivera-Pérez.

Email: sergioriveraperez@gmail.com

Author 3 - Damián Iglesias Gallego.

Email: diglesia@unex.es 